\title{
In Data We Trust: Proving Market Manipulation on the Tehran Stock Exchange
}

\author{
Noah Farhadi ${ }^{1} \&$ Hooshang Lahooti ${ }^{2}$ \\ ${ }^{1}$ Mammuttree ${ }^{\circledR}$ Analytics, Berlin and Macromedia University, Hamburg, Germany \\ 2 The University of Sydney, Sydney, Australia \\ Correspondence: Noah Farhadi, Mammuttree ${ }^{\circledR}$, Berlin, Germany. E-mail: info@mammuttree.com
}

Received: December 20, 2021

Accepted: February 17, 2022

Online Published: March 2, 2022

doi:10.5539/ijbm.v17n4p1

URL: https://doi.org/10.5539/ijbm.v17n4p1

\begin{abstract}
The Iranian financial markets play an essential role in the country's economic development. In 2019 and 2020 , ordinary traders were encouraged by the political authorities to invest in state-owned enterprises. Citizens who invested in Tehran Stock Exchange (TSE) indices routinely complain that the volatile market performance has wiped out their capital and savings. In this study, the reliability of intraday transaction data for 341 stocks listed on the TSE was examined. Our critical objective is to identify fraud on the TSE. The authors applied Benford's first and second digit laws to detect irregularities in financial data based on three goodness of fit tests. The authors found overwhelming evidence of the presence of market manipulation on the TSE. We found that 46 percent of the companies listed on the TSE did not adhere to the law of the first digit. A thorough analysis of compliance with the second digit revealed a similar pattern. Given the severe impact of trade restrictions imposed by the 2018 US sanctions and the substantial increase in Iran's public debt burden, the TSE has become a major source for offsetting the government's deficit by conducting IPOs of state-owned companies. Market manipulation in Iran appears to be motivated by the government's urgent need for fresh capital and its waste. It would be a common misconception to trust the TSE's data.
\end{abstract}

Keywords: Market Manipulation, Benford's Law, Forensic Analysis, Financial Markets, Data Science

\section{Introduction}

Financial markets are a captivating example of complexity in action: a complex, multi-layered system that evolves around decisions made by a multitude of individual traders who ceaselessly try to win in a global game. Middle Eastern traders are no exception. Every day, millions of transactions are executed in the region, helping both ordinary and institutional investors to succeed.

\subsection{Financial Market Manipulation}

It is generally agreed that financial markets facilitate efficient allocation of funds, maturity transformation, risk transfer, fair pricing, and selling of financial instruments. Investors seek to both maximize returns and minimize risks (Markowitz, 1952). Eugene Fama was the first who introduced the concept of "efficient markets" in his groundbreaking work, according to which information determines the price of assets (Fama, 1963, 1965a, 1965b, 1970). When investors buy (demand) and sell (supply) stocks, asset prices are generally set. The prevailing notion of efficient markets is founded on trust. As securities are claims on future distributions, investors participate in buy-side and sell-side transactions to the extent that they enjoy access to reliable data and are confident in future trades. Trust is the most important prerequisite for participation in trades.

Market manipulation is nothing new. It is "the unwarranted interference in the operation of ordinary market forces of supply and demand" (Lomnicka, 2010). It can take many forms: Dissemination of misinformation about assets, insider trading, or simulation of prices, rates, or trades to falsely imply higher demand for securities (see U.S. SEC 2020). With the advent of digital transformation and the emergence of online financial markets, it is now easier to disseminate and place fake orders to increase or depress securities prices (Finnerty, 2005).

According to Nasdaq (2021), spoofing typically involves placing large orders to feign high market demand, which may influence investors' behaviors. Another form of fraud is insider trading is front-running transactions that give investors early access to undisclosed and confidential information (Thomson Reuters, 2020). Conspiracy to deceive is a further type of market manipulation. It occurs whenever a fraudulent image of active trading is 
circulated. It is intrinsically motivated by the intent to maintain and maximize the market price at a predetermined level (Hauzen, 2021).

Fraud in the trading of stocks and financial products and other forms of market manipulation undermine the integrity of markets and can cause significant harm to investors. Lomnicka (2001) examined the ability of criminals to manipulate the financial markets in the United Kingdom. The author pointed to specific examples of financial market manipulation, such as misleading information to influence investment values or erroneous transaction volumes. There have been other attempts in the past literature to detect fraudulent financial data using statistical analyses (Luna, Palshikar, Apte, \& Bhattacharya, 2018; Stack, 2015; Beneish, 1999; Aggraval and Dhami, 2020; Karavardar, 2014; MacCarthy, 2017).

Karavardar (2014) studied the Istanbul Stock Exchange, applied Benford's law, and showed the compliance of the indices with the expected logarithmic law for the first and second digits and the first two first digits. A deviation from the logarithmic law does not always indicate fraud. Shengmin and Wenchao (2010) confirmed that the main indexes of the Shanghai Stock Exchange Composite Component Index followed the law. However, they provided empirical evidence of the influence of Chinese culture on trading patterns as observed by the abnormal absence of the number "4" in the Hong Kong stock market. In Chinese culture, the number four is widely viewed as a symbol of misfortune and bad luck. Non-compliance is also evident during periods of economic turmoil. While a study of S\&P 500 stock prices confirmed overall compliance, it also showed that market volatility or crash events resulted in non-compliance with the law (Corazza, Ellero, \& Zorzi, 2010).

\subsection{Tehran Stock Exchange}

The Tehran Stock Exchange (TSE) offers more than 350 financial instruments in primary and secondary submarkets. It plays a crucial role in Iran's economic development and enables state-owned enterprises to raise capital for future investments (Yazdani, 2008). Established in 1967, the market is a fast-moving market offering commodities, securities, corporate certificates, and bonds that can be traded immediately for cash (TSE History, 2020). The secondary submarket of the TSE includes financial securities issued by small and medium-sized enterprises (SMEs). Figure 1 provides an overview of the historical development of the TSE.

Economists have demonstrated that the decline in Iran's GDP and its weakening currency are attributable to the several sets of sanctions imposed on Iran (see Carswell, 1981), which came into effect in 1979. Since the sanctions have been in place, it has become a major political impediment to economic growth and development. Today, sanctions permeate all financial facets of Iran and take various forms, including trade bans, freezing Iranian assets, travel restrictions, and blocking all types of investment in commercial markets.

Furthermore, sanctions lead to market volatility and currency depreciation (Goudarzi 2014). Since the United States withdrew from the 2015 nuclear agreement on November 4, 2018, Iran's national currency IRR has depreciated significantly. For this reason, Iranian investors probably sought shelter to secure their weakening currency. Thus, they invested in TSE securities from 36 industries, such as hydrocarbons, automotive, agriculture, telecommunications, and services, especially financial services. According to a survey conducted by a progovernment student organization, the Iranian Student Polling Agency (ISPA), 20 percent of the Iranian population has somehow invested in TSE indices over the past two years. According to the same report, more than seventyseven percent of investors faced considerable loss, eight per percent made some profit, and 15 percent broke even. In April 2017, the index was at less than 100,000 points. Despite the severe impact of the U.S. sanctions and the related economic meltdown due to the COVID-19 outbreak, the index rose to 500,000 points by April 2018. The highly volatile market has recently exceeded the one-million-point threshold, representing an average annual growth rate of 1,040 percent in 2017 (Iran International News, 2021).

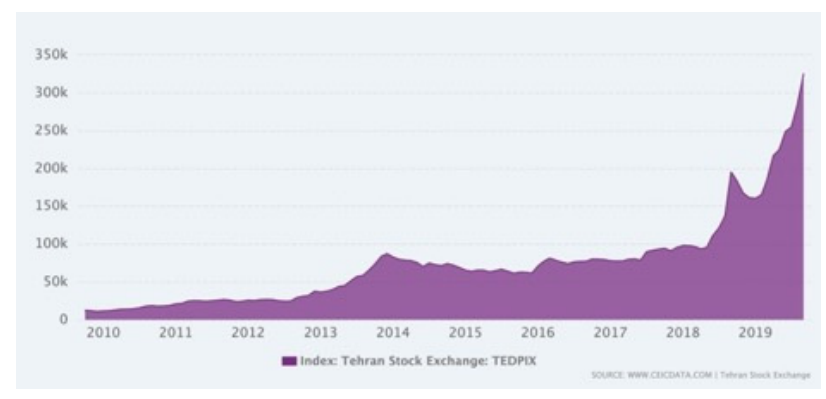

Figure 1. Tehran Stock Exchange (TSE) 
It is noteworthy that the political leadership in Iran has repeatedly encouraged households to invest in the primary stock market (see for example https:/www.leader.ir/en/content/24901/The-Leader\%E2\%80\%99s-message-onthe-occasion-of-Nowruz, last accessed: 19/9/2021). Simultaneously, the Iranian government contributed to the millionaire boom by flooding the TSE with an influx of taxpayers' money. The government poured $1 \%$ of the state fund (National Development Fund) into the index, followed by an additional tranche of 25 trillion rials, approximately $\$ 595.2$ million (Williams 2021). In the fall of 2020, the index exceeded two million points when the government began to sell its assets in the stock market and encouraged households to engage in investments. The higher the market rose, the more people saw the TSE as a common way to make money. However, the market soon fell by approximately half its value. After recouping some of its losses, it stands at around 1.3 million points (Iran International News, 2021). The 13-fold increase over the four years did not mean that people made much profit, as many entered the market too late. Figure 1 shows the TSE trend between 2010 and 2021(TSE, 2021b). It is noteworthy that the number of millionaires in Iran has skyrocketed in recent years, contrary to general economic trends. In 2020, the total number of High-Net-Worth Individuals (or HNWI) grew by $21.6 \%$ in Iran, far above the global average of $6.3 \%$ (Khaasteh, 2021). In 2021 alone, the total wealth of these U.S. dollar millionaires increased by $24.3 \%$.

The central question of this study is: Did the Iranian government fill its empty coffers by manipulating the financial market?

\section{Methods}

\subsection{Benford's Law}

It is common knowledge that natural numbers follow expected frequencies. The so-called logarithmic law, or Benford's law (BL), was first invented by the Canadian American mathematician Newcomb (1881). BL distinguishes natural from fictitious figures and provides empirical evidence of data manipulation if any, (Ravenda, Valencia-Silva, Argiles-Bosch \& Garcia-Blandon, 2018). In this context, BL is instrumental in detecting anomalies and fraud (Nigrini, 2000) and was previously operationalized to identify fraudulent financial data.

Several studies have addressed market manipulation and have applied statistical techniques to detect such attempts. These studies unanimously used the numerical frequency law of first digits, also known as Benford's law (BL). In addition to the order of the first digit, other frequencies can also be determined for the second digit. The core idea refers to the frequencies of the first and second digits in naturally generated datasets according to Equations 1 and 2 (Newcomb, 1981; Benford, 1938):

$$
\begin{gathered}
\mathrm{P}(\mathrm{d})=\log _{-} 10(1+1 / \mathrm{d}), \mathrm{d} \in\{1,2,3, \ldots, 9\} \\
\mathrm{P}(\mathrm{d})=\sum_{j=1}^{9} \log _{-} 10[1+1 /(10 j+d)], \mathrm{d} \in\{0,1,2,3, \ldots, 9\}
\end{gathered}
$$

\begin{tabular}{|c|c|c|c|c|c|c|c|c|c|c|c|}
\hline \multicolumn{12}{|c|}{ Second significant digit } \\
\hline & $\mathbf{0}$ & 1 & 2 & 3 & 4 & 5 & 6 & 7 & 8 & 9 & $\approx \Sigma$ \\
\hline 1 & 0.041 & 0.038 & 0.035 & 0.032 & 0.030 & 0.028 & 0.026 & 0.025 & 0.023 & 0.022 & 0.301 \\
\hline 2 & 0.021 & 0.020 & 0.019 & 0.018 & 0.018 & 0.017 & 0.016 & 0.016 & 0.015 & 0.015 & 0.176 \\
\hline 3 & 0.014 & 0.014 & 0.013 & 0.013 & 0.013 & 0.012 & 0.012 & 0.012 & 0.011 & 0.011 & 0.125 \\
\hline 4 & 0.011 & 0.010 & 0.010 & 0.010 & 0.010 & 0.010 & 0.009 & 0.009 & 0.009 & 0.009 & 0.097 \\
\hline 5 & 0.009 & 0.008 & 0.008 & 0.008 & 0.008 & 0.008 & 0.008 & 0.008 & 0.007 & 0.007 & 0.079 \\
\hline 6 & 0.007 & 0.007 & 0.007 & 0.007 & 0.007 & 0.007 & 0.007 & 0.006 & 0.006 & 0.006 & 0.067 \\
\hline 7 & 0.006 & 0.006 & 0.006 & 0.006 & 0.006 & 0.006 & 0.006 & 0.006 & 0.006 & 0.005 & 0.058 \\
\hline 8 & 0.005 & 0.005 & 0.005 & 0.005 & 0.005 & 0.005 & 0.005 & 0.005 & 0.005 & 0.005 & 0.051 \\
\hline $\mathbf{0}$ & 0.005 & 0.005 & 0.005 & 0.005 & 0.005 & 0.005 & 0.005 & 0.004 & 0.004 & 0.004 & 0.046 \\
\hline$\approx \Sigma$ & 0.120 & 0.114 & 0.109 & 0.104 & 0.100 & 0.097 & 0.093 & 0.090 & 0.088 & 0.085 & \\
\hline
\end{tabular}

Table 1: First and second digits' joint and marginal distributions

Table 1 summarizes frequencies of the first and second digits. The first- and second-digit tests are high-level adequacy tests used in a complementary manner to assess whether the dataset is adequate. According to Benford's law for the first digit (1BL), the numbers one to nine follow a certain logarithmic distribution: $30.1 \%$ for one, $17.6 \%$ for two, $12.5 \%$ for three, $9.7 \%$ for four, $7.9 \%$ for five, $6.7 \%$ for six, $5.8 \%$ for seven, $5.1 \%$ for eight, and $4.6 \%$ for nine [20]; for Benford's law for two digits (2BL), the frequencies are distributed from zero to nine: $12 \%$ for zero, 
$11.4 \%$ for one, $10.9 \%$ for two, $10.4 \%$ for three, $10.0 \%$ for four, $9.7 \%$ for five, $9.3 \%$ for six, $9.0 \%$ for seven, $8.8 \%$ for eight, and $8.5 \%$ for nine (Nigrini, 1999, Newcomb, 1881).

The observed frequencies are likely to deviate from the BL distributions in an artificially generated dataset. Benfordness - compliance with the law - can be measured by applying financial data with a geometric trend and is characterized by the absence of minima and maxima. BL is a common practice and has been widely used in various disciplines, such as financial markets (Karavardar, 2014), epidemiology (Sambridge \& Jackson, 2020; Farhadi, 2020; Farhadi \& Lahooti, 2021a, 2021b), finance, and accounting (see Durtschi, Hillison, \& Pacini, 2004), political science (Roukema, 2014), and compliance (Deleanu, 2017). The body of knowledge provides several tests to assess such agreement. Three standard techniques are well-known and widely used: the Kuiper test, chisquare test, and mean average deviation (MAD) (see Farhadi \& Lahooti, 2021a).

The Kuiper statistic is a nonparametric test of discrete data. It is a modification of the Kolmogorov-Smirnov test. It quantifies the distance between the empirical distribution of samples of observations and the cumulative distribution of first-and second-digit frequencies [9]. The two summed parts are $D_{n}^{+}$and $D_{n}^{-}$is calculated as $D_{n}^{+}=\operatorname{Max}[F n(x)-F 0(x)]$ and $D_{n}^{-}=\operatorname{Max}[F 0(x)-F n(x)]$, where $F n$ is the cumulative observed distribution and $F_{-} 0$ is the cumulative Benford distribution. Nonparametric tests are distribution-free and more powerful when small sample size [25]. The Kuiper test is calculated as follows (see Equation 3):

$$
K=\left(D_{n}^{+}+D_{n}^{-}\right) \times[\sqrt{n}+0.155+0.24 / \sqrt{n}]
$$

Another popular approach is Pearson's chi-square test $\left(\chi^{\wedge} 2\right)$ with the confirmatory null hypothesis that the distribution of the first digit must match Benford's frequency curve [12]. The chi-square test is sensitive to the sample size and is not recommended for inference when the dataset exceeds 5,000 observations $[22,26]$. The chisquared statistic uses the expected number of observations. If the sample size is too large, the null hypothesis is rejected even if there is no significant difference between the actual and expected subsets. The chi-squared test was calculated as follows (see Equation 4):

$$
\chi^{2}=\sum_{I=1}^{9}\left(\tilde{p}_{i}-p_{i}\right)^{2} / p_{i}
$$

In this study, the mean absolute deviation (MAD) technique was used, less dependent on sample size. Equation 5 shows the MAD calculations for the observed and expected frequencies. $k$ is the number of leading digit bins. $O_{\text {. }}$. and $E_{. I}$ are the observed and expected frequencies, respectively. A MAD $>0.015$ indicates a lack of agreement with the law (Nigrini, 2018).

$$
M A D=1 / k \times \sum_{i=1}^{k}\left|O_{i}-E_{i}\right|
$$

Furthermore, we operationalized all goodness-of-fit tests based on a significance level of 0.05 . We tested all shares of listed companies in Iran for the following hypotheses.

H0: The frequency of the volume of $J i$ adheres to BL, where $J i$ stands for a specific security listed on the Tehran Stock Exchange.

\subsection{Sampling of Data}

We collected data on the number of intraday transactions published by the TSE from 21/3/2019 to 8/9/2021. The original datasets used in this study were obtained from the TSE website. The sample consisted of 176,618 integers ( $n$ or the sample size). Intermediaries, who disseminate information on financial instruments, can centrally control and theoretically change the number of intraday transactions. As mentioned earlier, changes in the number of transactions may signal higher demand and improve the attractiveness of traded securities. For each security, we compiled a sample of more than 100 observations, that is, the minimum number of intraday transactions, which can be considered an acceptable size for the application of Benford's law, even though the minimum threshold is not specified in the literature. Measuring compliance with the BL law is only helpful if the sample size is not too small. Small datasets may erroneously deviate from the expected logarithmic law and affect the reliability of the results. It was assumed that BL compliance increase as the sample size gets larger.

\section{Results}

\subsection{Benfordness}

To explore the BL agreement of data, six goodness-of-fit tests were conducted in this study: the Kuiper test, chisquare goodness-of-fit test, and MAD for the first and second digits of intraday transactions. Forty-one securities were included in the primary sample, with an average number of observations of $n=536$, resulting in a subset of 330 stocks with over 100 records. This selection was necessary to perform reliable tests to assess the Benfordness. The MAD, Kuiper, and Chi-square tests quantified the distance between the reported and referenced Benford 
distributions.

Table 2. Critical values of the empirical tests

\begin{tabular}{lllllll}
\hline Alpha level & Chi-Square & & Kuiper & MAD & 2nd digit \\
& 1st digit & 2nd digit & 1st digit & 2nd digit & 1st digit & 0.006 \\
\hline 0.01 & 13.36 & 14.68 & 1.62 & 1.62 & 0.012 & 0.010 \\
0.05 & 15.51 & 16.92 & 1.75 & 1.75 & 0.015 & 0.012 \\
0.1 & 20.09 & 21.67 & 2 & 2 & \\
\hline
\end{tabular}

The results are summarized in Tables 3-5, including list of financial products that did not conform to BL at an 0.1 alpha level. These assets are marked and highlighted in red. One hundred forty-eight assets did not meet the BL compliance requirements based on at least two goodness-of-fit tests for the first and second digits; thus, we reject the null hypothesis, suggesting market manipulation of the TSE. Looking at the Kuiper statistics for first- and second-digit compliance, nine assets with a total market capitalization of IRR 998,270,000 do not meet BL compliance requirements. A similar analysis of the MAD statistics resulted in a subset of 63 assets that did not meet the Benfordness thresholds. One hundred thirty-three securities did not pass the first-digit chi-squared test for adequacy. Figure 2 shows the frequency of the first leading digits of the most significant violations.

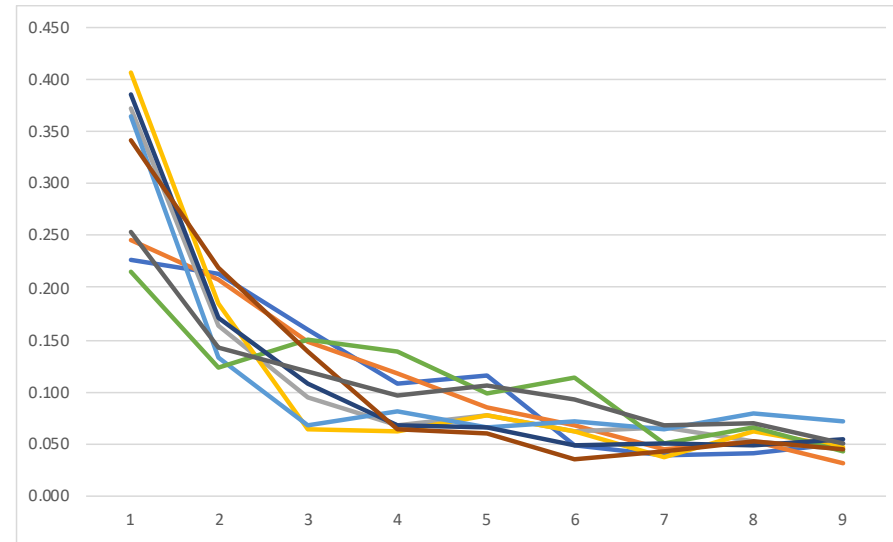

Figure 2: The most common violations of Benford's Law

Table 3: Results of the statistical and BL goodness of fit tests

\begin{tabular}{|c|c|c|c|c|c|c|c|c|c|}
\hline Name & $\mathbf{N}$ & Volatility & Avg. Return & Kuiper 1D & Kuiper 2D & MAD 1D & MAD 2D & CHIS 1D & CHIS 2D \\
\hline Abadan power generation & 257 & 0.951 & 1.057 & 0.907 & 0.865 & 0.017 & 0.017 & 8.357 & 9.944 \\
\hline Abssal & 562 & 0.179 & 1.003 & 2.689 & 0.973 & 0.020 & 0.008 & 25.895 & 4.816 \\
\hline Alborz Cable & 558 & 0.173 & 1.005 & 4.262 & 1.053 & 0.032 & 0.013 & 58.067 & 10.681 \\
\hline Alborz Insurance & 518 & 0.187 & 1.006 & 1.854 & 1.297 & 0.015 & 0.014 & 14.866 & 13.806 \\
\hline Alborz Investment (Holding & 554 & 0.183 & 1.005 & 1.370 & 2.046 & 0.012 & 0.015 & 8.298 & 14.198 \\
\hline Alborz medicine & 558 & 0.313 & 0.012 & 1.494 & 1.210 & 0.012 & 0.010 & 9.607 & 12.236 \\
\hline Aluminum Iran & 558 & 0.172 & 1.004 & 1.605 & 1.053 & 0.013 & 0.009 & 11.851 & 5.388 \\
\hline Alumrad & 513 & 0.191 & 1.006 & 1.882 & 0.909 & 0.015 & 0.011 & 14.187 & 8.368 \\
\hline Alvand Tile and Ceramic & 557 & 0.201 & 1.004 & 2.554 & 1.232 & 0.020 & 0.011 & 21.133 & 6.816 \\
\hline Amin Capital & 244 & 0.153 & 1.002 & 2.473 & 1.371 & 0.028 & 0.019 & 27.430 & 11.280 \\
\hline Arak Construction Machine & 560 & 0.188 & 1.003 & 4.784 & 0.563 & 0.036 & 0.007 & 75.529 & 2.644 \\
\hline Asia Insurance & 545 & 0.180 & 1.005 & 1.120 & 1.705 & 0.009 & 0.017 & 4.797 & 19.641 \\
\hline Automobile Engineering & 518 & 0.182 & 1.007 & 3.388 & 2.207 & 0.028 & 0.016 & 35.630 & 12.888 \\
\hline Axis makers of Iran & 560 & 0.190 & 1.004 & 2.137 & 0.830 & 0.016 & 0.009 & 17.763 & 6.561 \\
\hline
\end{tabular}




\begin{tabular}{|c|c|c|c|c|c|c|c|c|c|}
\hline Name & $\mathbf{N}$ & Volatility & Avg. Return & Kuiper 1D & Kuiper 2D & MAD 1D & MAD 2D & CHIS 1D & CHIS 2D \\
\hline Azar Refractory Products & 552 & 0.195 & 1.005 & 3.113 & 1.045 & 0.025 & 0.012 & 33.719 & 11.745 \\
\hline Bafgh Mines & 532 & 0.180 & 1.004 & 2.362 & 0.854 & 0.018 & 0.013 & 18.929 & 9.812 \\
\hline Bandar Abbas oil refining & 508 & 0.199 & 1.004 & 1.473 & 0.808 & 0.012 & 0.009 & 9.119 & 5.451 \\
\hline Bank Mellat Capital & 322 & 0.371 & 1.011 & 0.900 & 0.900 & 0.010 & 0.011 & 5.041 & 6.415 \\
\hline Bank of Economics & 549 & 0.180 & 1.003 & 1.540 & 1.071 & 0.015 & 0.011 & 13.668 & 8.085 \\
\hline Bank Saderat Iran & 546 & 0.178 & 1.004 & 3.228 & 1.049 & 0.025 & 0.015 & 29.717 & 17.022 \\
\hline Bank Sina & 555 & 0.184 & 1.004 & 1.541 & 1.453 & 0.013 & 0.012 & 12.426 & 11.323 \\
\hline Banks are entrepreneurs & 533 & 0.163 & 1.004 & 1.667 & 0.911 & 0.013 & 0.008 & 11.540 & 5.882 \\
\hline Barakat Pharmaceutical Group & 549 & 0.191 & 1.007 & 0.504 & 0.732 & 0.008 & 0.007 & 4.965 & 3.520 \\
\hline Barez Industrial Group & 563 & 0.181 & 1.004 & 2.204 & 1.515 & 0.019 & 0.010 & 25.013 & 9.409 \\
\hline Behnoosh Iran & 557 & 0.179 & 1.005 & 2.099 & 0.563 & 0.016 & 0.006 & 19.138 & 1.854 \\
\hline Behshahr Industrial Dev. Holding & 572 & 0.176 & 1.004 & 2.246 & 0.686 & 0.017 & 0.008 & 22.322 & 3.175 \\
\hline Behshahr Industrial Group of Iran & 570 & 0.173 & 1.004 & 0.980 & 1.650 & 0.010 & 0.011 & 11.406 & 9.850 \\
\hline Behshahr Industry & 561 & 0.182 & 1.004 & 1.223 & 1.181 & 0.014 & 0.010 & 9.517 & 8.117 \\
\hline Bimeh Dana & 562 & 0.182 & 1.004 & 1.427 & 0.818 & 0.011 & 0.007 & 7.231 & 5.133 \\
\hline Biscuit Georgia & 494 & 0.185 & 1.004 & 0.971 & 1.444 & 0.011 & 0.012 & 7.567 & 13.846 \\
\hline Bootan Industrial Group & 566 & 0.169 & 1.004 & 1.941 & 1.318 & 0.015 & 0.010 & 14.884 & 10.618 \\
\hline Brake pads & 548 & 0.191 & 1.005 & 1.409 & 0.756 & 0.013 & 0.012 & 12.465 & 6.011 \\
\hline Calcium & 558 & 0.177 & 1.004 & 2.112 & 1.155 & 0.016 & 0.009 & 18.175 & 6.640 \\
\hline Campus Investment & 577 & 0.171 & 1.004 & 1.485 & 1.042 & 0.012 & 0.008 & 9.575 & 3.082 \\
\hline Capital of hope & 562 & 0.171 & 1.004 & 1.570 & 0.890 & 0.012 & 0.007 & 9.928 & 5.895 \\
\hline Car axle production & 521 & 0.175 & 1.005 & 2.606 & 0.627 & 0.021 & 0.008 & 25.061 & 4.042 \\
\hline Carbon Iran & 567 & 0.177 & 1.004 & 4.006 & 0.668 & 0.030 & 0.010 & 42.749 & 7.925 \\
\hline Cartoon of Iran & 513 & 0.181 & 1.003 & 3.089 & 1.546 & 0.024 & 0.014 & 27.989 & 18.910 \\
\hline Caspian Sea & 556 & 0.188 & 1.005 & 2.293 & 0.795 & 0.018 & 0.007 & 20.303 & 4.698 \\
\hline Caspian Sea & 545 & 0.169 & 1.005 & 0.738 & 0.929 & 0.013 & 0.009 & 14.548 & 6.999 \\
\hline Cement & 561 & 0.175 & 1.005 & 1.422 & 1.972 & 0.015 & 0.016 & 18.963 & 22.290 \\
\hline Cement Arta Ardabil & 557 & 0.175 & 1.004 & 1.233 & 1.778 & 0.012 & 0.016 & 8.333 & 11.203 \\
\hline Cement Industry Dev. Investment & 572 & 0.168 & 1.004 & 2.819 & 0.913 & 0.021 & 0.013 & 23.403 & 14.858 \\
\hline Century Chemistry Phenomenon & 345 & 0.250 & 0.008 & 1.469 & 0.568 & 0.014 & 0.009 & 10.284 & 4.023 \\
\hline Ceramics of Ardakan industry & 532 & 0.188 & 1.005 & 4.038 & 0.799 & 0.031 & 0.008 & 69.862 & 3.465 \\
\hline Chemical Medicine & 559 & 0.178 & 1.003 & 3.782 & 1.621 & 0.030 & 0.012 & 56.045 & 11.380 \\
\hline Chin-Chin culture and industry & 516 & 0.174 & 1.005 & 0.904 & 1.287 & 0.015 & 0.013 & 16.173 & 15.683 \\
\hline Civilization and development of Persia & 546 & 0.182 & 1.005 & 0.933 & 0.884 & 0.007 & 0.011 & 3.260 & 7.931 \\
\hline Coal Stone Tabas & 558 & 0.176 & 1.004 & 4.805 & 0.846 & 0.037 & 0.008 & 77.891 & 5.292 \\
\hline Combine construction in Iran & 562 & 0.174 & 1.004 & 2.175 & 1.198 & 0.017 & 0.011 & 18.592 & 9.731 \\
\hline Commercial Bank & 530 & 0.181 & 1.004 & 0.999 & 0.992 & 0.009 & 0.010 & 5.151 & 9.119 \\
\hline Construction structure & 486 & 0.161 & 1.005 & 0.865 & 0.629 & 0.012 & 0.005 & 8.255 & 2.995 \\
\hline Damavand Atieh Investment & 567 & 0.176 & 1.004 & 2.907 & 1.493 & 0.022 & 0.012 & 28.944 & 10.909 \\
\hline Damavand Mining & 477 & 0.372 & 0.028 & 1.381 & 1.721 & 0.011 & 0.014 & 6.577 & 12.350 \\
\hline Daroopakhsh & 550 & 0.261 & 0.009 & 2.725 & 2.506 & 0.021 & 0.019 & 24.843 & 18.809 \\
\hline Dasht Morghab & 423 & 0.415 & 0.035 & 1.112 & 0.934 & 0.010 & 0.014 & 8.693 & 11.093 \\
\hline Dashtestan Cement Industries & 540 & 0.151 & 1.006 & 1.580 & 1.558 & 0.012 & 0.012 & 20.092 & 11.299 \\
\hline Data Processing Iran & 569 & 0.176 & 0.004 & 3.110 & 0.987 & 0.023 & 0.009 & 25.724 & 6.779 \\
\hline
\end{tabular}




\begin{tabular}{|c|c|c|c|c|c|c|c|c|c|}
\hline Name & $\mathbf{N}$ & Volatility & Avg. Return & Kuiper 1D & Kuiper 2D & MAD 1D & MAD 2D & CHIS 1D & CHIS 2D \\
\hline Desert tire & 562 & 0.179 & 1.004 & 1.412 & 1.510 & 0.016 & 0.011 & 21.462 & 4.535 \\
\hline Detergent Industry Mgmt, Behshahr & 575 & 0.173 & 1.003 & 2.122 & 1.145 & 0.016 & 0.010 & 18.419 & 5.305 \\
\hline Development and development of hope & 234 & 0.239 & 1.004 & 1.870 & 1.032 & 0.022 & 0.019 & 13.901 & 9.589 \\
\hline Development of Iranian surface mines & 567 & 0.180 & 1.004 & 1.066 & 0.970 & 0.008 & 0.008 & 6.204 & 5.801 \\
\hline Development of mines and metals & 518 & 0.171 & 1.004 & 1.088 & 0.515 & 0.009 & 0.007 & 4.640 & 2.551 \\
\hline Development system & 532 & 0.181 & 1.003 & 1.557 & 0.941 & 0.012 & 0.008 & 10.334 & 5.239 \\
\hline Driving Force & 544 & 0.261 & 1.007 & 2.258 & 1.054 & 0.017 & 0.008 & 22.202 & 6.033 \\
\hline Drugs & 537 & 0.452 & 0.046 & 1.349 & 0.864 & 0.012 & 0.010 & 13.913 & 6.328 \\
\hline Dudeh Industrial Pars & 567 & 0.227 & 0.005 & 4.207 & 0.987 & 0.031 & 0.009 & 59.193 & 4.445 \\
\hline East Cement & 567 & 0.182 & 1.004 & 2.694 & 0.831 & 0.023 & 0.007 & 28.843 & 3.269 \\
\hline Easy to pay Persian & 573 & 0.171 & 1.002 & 1.769 & 1.474 & 0.015 & 0.011 & 17.623 & 9.619 \\
\hline Electric car east & 571 & 0.212 & 0.998 & 3.425 & 1.168 & 0.026 & 0.009 & 38.543 & 6.451 \\
\hline Electric car east & 569 & 0.183 & 1.004 & 3.425 & 1.168 & 0.026 & 0.009 & 38.543 & 6.451 \\
\hline Energy 31 & 213 & 3.545 & 1.862 & 2.636 & 0.742 & 0.034 & 0.014 & 27.955 & 5.726 \\
\hline Energy Capital Investment & 502 & 0.191 & 1.003 & 0.938 & 1.346 & 0.009 & 0.013 & 6.929 & 8.396 \\
\hline Expansion of Iran Khodro Investment & 545 & 0.239 & 1.007 & 1.819 & 0.800 & 0.014 & 0.009 & 15.309 & 6.751 \\
\hline Expansion of Persian oil and gas & 574 & 0.170 & 1.004 & 1.780 & 2.155 & 0.013 & 0.015 & 14.845 & 15.103 \\
\hline Extension Industries Serv. Agriculture & 554 & 0.179 & 1.005 & 1.931 & 0.808 & 0.015 & 0.008 & 12.921 & 5.130 \\
\hline Factories Razi glass & 522 & 0.181 & 1.005 & 1.587 & 1.047 & 0.015 & 0.013 & 12.308 & 11.962 \\
\hline Factories of drug distribution & 564 & 0.186 & 0.005 & 1.567 & 0.933 & 0.012 & 0.008 & 14.248 & 3.901 \\
\hline Faiber Iran & 552 & 0.262 & 0.007 & 1.360 & 0.783 & 0.011 & 0.006 & 8.579 & 1.774 \\
\hline Fajr Energy Persian Gulf & 563 & 0.165 & 1.003 & 2.602 & 0.855 & 0.020 & 0.009 & 28.909 & 6.662 \\
\hline Fans I & 548 & 0.197 & 1.004 & 2.136 & 1.291 & 0.016 & 0.015 & 24.401 & 10.213 \\
\hline Fans II & 543 & 0.268 & 0.009 & 1.936 & 1.470 & 0.015 & 0.011 & 20.071 & 9.013 \\
\hline Fars and Khuzestan Cement & 566 & 0.188 & 1.005 & 2.585 & 1.601 & 0.019 & 0.016 & 23.662 & 12.277 \\
\hline February Investment & 568 & 0.181 & 1.005 & 1.517 & 1.273 & 0.012 & 0.009 & 9.485 & 7.929 \\
\hline Ferro Silica Iran & 532 & 0.175 & 0.004 & 0.790 & 0.752 & 0.009 & 0.007 & 5.666 & 4.725 \\
\hline Food control & 536 & 0.177 & 1.001 & 2.407 & 1.213 & 0.019 & 0.012 & 22.738 & 8.380 \\
\hline Fund investment Retirement & 574 & 0.166 & 1.004 & 1.520 & 1.281 & 0.011 & 0.011 & 10.442 & 6.822 \\
\hline Ghadir Investment (Holding) & 578 & 0.174 & 1.004 & 2.903 & 0.674 & 0.022 & 0.008 & 34.073 & 5.037 \\
\hline Ghologhozan & 535 & 0.460 & 0.050 & 1.254 & 1.167 & 0.013 & 0.013 & 11.351 & 9.257 \\
\hline Gholtash & 572 & 0.173 & 1.003 & 2.678 & 1.224 & 0.021 & 0.011 & 24.089 & 8.384 \\
\hline Glass \& Gas & 564 & 0.178 & 1.004 & 3.074 & 1.787 & 0.023 & 0.015 & 42.696 & 15.679 \\
\hline Group of Crafts in the city of Iran & 474 & 0.181 & 1.005 & 2.014 & 1.857 & 0.016 & 0.016 & 11.475 & 15.576 \\
\hline Healing Pharma investment & 567 & 0.173 & 1.004 & 1.275 & 1.384 & 0.010 & 0.013 & 6.098 & 12.730 \\
\hline Housing investment & 572 & 0.179 & 1.004 & 2.209 & 1.297 & 0.017 & 0.013 & 17.403 & 14.307 \\
\hline Industrial Group & 549 & 0.190 & 1.005 & 1.440 & 1.261 & 0.011 & 0.011 & 7.253 & 7.768 \\
\hline Industrial pasta & 250 & 1.566 & 1.156 & 2.270 & 1.047 & 0.026 & 0.019 & 19.331 & 12.076 \\
\hline Industries of Azar & 446 & 0.185 & 1.006 & 3.051 & 0.767 & 0.026 & 0.009 & 30.011 & 5.243 \\
\hline Industries of paper making & 533 & 0.183 & 1.005 & 2.497 & 0.826 & 0.019 & 0.010 & 31.317 & 10.372 \\
\hline Industries of welding & 540 & 0.168 & 1.008 & 1.491 & 1.536 & 0.012 & 0.011 & 12.136 & 9.753 \\
\hline Industry Ama & 563 & 0.173 & 1.004 & 0.913 & 0.852 & 0.007 & 0.013 & 6.844 & 12.695 \\
\hline Industry \& Trade Dev. Investment & 573 & 0.179 & 1.004 & 1.307 & 1.191 & 0.010 & 0.012 & 9.542 & 8.698 \\
\hline Informatics Services & 558 & 0.167 & 1.002 & 1.369 & 1.010 & 0.010 & 0.007 & 5.879 & 4.127 \\
\hline
\end{tabular}




\begin{tabular}{|c|c|c|c|c|c|c|c|c|c|}
\hline Name & $\mathbf{N}$ & Volatility & Avg. Return & Kuiper 1D & Kuiper 2D & MAD 1D & MAD 2D & CHIS 1D & CHIS 2D \\
\hline International Dev. of Construction & 560 & 0.182 & 1.003 & 1.611 & 1.275 & 0.012 & 0.010 & 10.865 & 9.400 \\
\hline International Products of Persia & 556 & 0.183 & 1.004 & 1.995 & 1.430 & 0.019 & 0.014 & 25.648 & 15.432 \\
\hline Investment Capital & 564 & 0.177 & 1.004 & 1.650 & 1.183 & 0.014 & 0.012 & 10.603 & 10.345 \\
\hline Investment Development & 577 & 0.166 & 1.004 & 1.073 & 1.320 & 0.008 & 0.009 & 6.893 & 6.714 \\
\hline Investment in Petrochemical Industries & 571 & 0.181 & 1.004 & 4.515 & 0.675 & 0.034 & 0.008 & 55.642 & 6.991 \\
\hline Investment Dev. of Azerbaijan & 542 & 0.187 & 1.006 & 0.519 & 1.315 & 0.007 & 0.011 & 4.899 & 11.593 \\
\hline Investment in the Insurance Industry & 562 & 0.171 & 1.002 & 3.123 & 1.057 & 0.024 & 0.011 & 32.363 & 11.344 \\
\hline Investment in the Oil Industry & 575 & 0.176 & 1.003 & 1.438 & 0.908 & 0.011 & 0.006 & 7.551 & 3.527 \\
\hline Investment in Tocafulad & 548 & 0.180 & 1.005 & 3.830 & 1.095 & 0.030 & 0.009 & 51.213 & 6.154 \\
\hline Investment of Parsh Tosheh & 546 & 0.174 & 1.004 & 2.226 & 1.287 & 0.017 & 0.012 & 15.292 & 5.757 \\
\hline Investment of Sepah & 565 & 0.166 & 1.004 & 1.172 & 1.115 & 0.012 & 0.012 & 11.280 & 8.502 \\
\hline Investment in Construction, Iran & 533 & 0.191 & 1.006 & 1.672 & 1.488 & 0.013 & 0.011 & 11.593 & 7.589 \\
\hline Iran Tire & 542 & 0.184 & 1.006 & 3.123 & 1.894 & 0.024 & 0.013 & 28.294 & 14.593 \\
\hline Iran Alloy Steel & 546 & 0.328 & 0.016 & 1.261 & 1.477 & 0.016 & 0.010 & 16.539 & 10.278 \\
\hline Iran Commodity Exchange & 520 & 0.189 & 1.006 & 1.863 & 1.452 & 0.015 & 0.010 & 19.388 & 5.527 \\
\hline Iran Credit Investment & 574 & 0.173 & 1.002 & 0.978 & 1.017 & 0.009 & 0.010 & 7.128 & 7.095 \\
\hline Iran Industrial Development Group & 563 & 0.178 & 1.004 & 0.920 & 0.993 & 0.007 & 0.009 & 4.876 & 8.319 \\
\hline Iran Khodro & 526 & 0.236 & 1.006 & 1.502 & 1.117 & 0.017 & 0.012 & 18.330 & 9.182 \\
\hline Iran Kish credit card & 552 & 0.173 & 1.004 & 1.351 & 1.105 & 0.015 & 0.011 & 12.393 & 11.075 \\
\hline Iran Manganese Mine & 566 & 0.177 & 1.005 & 3.827 & 2.410 & 0.029 & 0.018 & 48.829 & 25.694 \\
\hline Iran Mineral Processing & 546 & 0.437 & 0.041 & 2.907 & 1.144 & 0.024 & 0.014 & 27.257 & 18.004 \\
\hline Iran Mobile Communications Co. & 557 & 0.162 & 1.003 & 2.395 & 0.683 & 0.018 & 0.009 & 20.254 & 7.020 \\
\hline Iran Numbers & 554 & 0.181 & 1.003 & 2.672 & 1.307 & 0.020 & 0.011 & 28.130 & 4.565 \\
\hline Iran Pharma & 552 & 0.193 & 1.006 & 1.557 & 0.787 & 0.017 & 0.009 & 13.923 & 8.534 \\
\hline Iran Pipe and Machine Development & 515 & 0.182 & 1.003 & 2.965 & 0.855 & 0.023 & 0.012 & 30.726 & 11.281 \\
\hline Iran Pump & 557 & 0.176 & 1.004 & 1.513 & 1.523 & 0.011 & 0.011 & 7.954 & 8.897 \\
\hline Iran Telecommunication & 531 & 0.204 & 1.004 & 1.920 & 0.968 & 0.016 & 0.008 & 19.389 & 4.946 \\
\hline Iran Transfo & 530 & 0.185 & 1.003 & 0.842 & 1.413 & 0.009 & 0.010 & 3.955 & 7.801 \\
\hline Iran, Yasatiro Rabar & 544 & 0.180 & 1.004 & 2.164 & 0.887 & 0.017 & 0.006 & 15.361 & 4.114 \\
\hline Iranian crying industry & 544 & 0.188 & 1.004 & 2.376 & 1.108 & 0.018 & 0.009 & 17.450 & 8.095 \\
\hline Iranian glass wool & 542 & 0.173 & 1.005 & 1.414 & 1.191 & 0.011 & 0.015 & 6.832 & 11.109 \\
\hline Iranian injection products & 567 & 0.168 & 0.003 & 1.656 & 0.463 & 0.014 & 0.006 & 12.509 & 3.074 \\
\hline Iranian Leasing & 542 & 0.179 & 1.005 & 1.799 & 0.530 & 0.014 & 0.007 & 12.621 & 2.577 \\
\hline Iranian National Capital Investment & 555 & 0.165 & 1.005 & 1.195 & 1.005 & 0.011 & 0.011 & 7.213 & 9.764 \\
\hline Iranian refractory products & 549 & 0.189 & 1.003 & 3.004 & 1.186 & 0.023 & 0.009 & 25.738 & 6.793 \\
\hline Iranian Soil Industry & 557 & 0.161 & 1.005 & 2.360 & 1.199 & 0.018 & 0.010 & 25.037 & 5.764 \\
\hline Irka Part Industry & 539 & 0.193 & 1.005 & 4.520 & 0.650 & 0.035 & 0.007 & 71.789 & 6.316 \\
\hline Isfahan Mobarakeh Steel & 560 & 0.297 & 0.011 & 1.234 & 1.749 & 0.011 & 0.012 & 7.060 & 13.618 \\
\hline Isfahan Oil Refining & 544 & 0.180 & 1.004 & 3.179 & 1.701 & 0.024 & 0.013 & 31.805 & 12.570 \\
\hline Kamal & 555 & 0.202 & 1.005 & 2.956 & 0.835 & 0.024 & 0.011 & 29.311 & 8.828 \\
\hline Kaveh Steel South Kish & 560 & 0.178 & 1.004 & 1.287 & 1.393 & 0.012 & 0.009 & 9.065 & 5.729 \\
\hline Kermanshah Petrochemical Industries & 576 & 0.163 & 1.004 & 0.767 & 1.061 & 0.008 & 0.011 & 5.189 & 7.920 \\
\hline Keshavarzi & 548 & 0.183 & 1.004 & 2.904 & 0.652 & 0.022 & 0.008 & 26.038 & 4.454 \\
\hline Kharazmi Investment & 548 & 0.194 & 1.005 & 3.650 & 1.764 & 0.028 & 0.013 & 41.206 & 13.673 \\
\hline
\end{tabular}




\begin{tabular}{|c|c|c|c|c|c|c|c|c|c|}
\hline Name & $\mathbf{N}$ & Volatility & Avg. Return & Kuiper 1D & Kuiper 2D & MAD 1D & MAD 2D & CHIS 1D & CHIS 2D \\
\hline Khorasan Petrochemical & 562 & 0.160 & 1.004 & 1.412 & 1.009 & 0.017 & 0.011 & 16.616 & 9.983 \\
\hline Khorasan Steel & 565 & 0.251 & 0.006 & 1.328 & 1.279 & 0.015 & 0.011 & 17.851 & 11.339 \\
\hline Khuzestan Cement & 563 & 0.180 & 1.005 & 2.127 & 0.926 & 0.016 & 0.008 & 21.451 & 5.190 \\
\hline Kowsar Agricultural Investment & 345 & 0.808 & 1.037 & 1.459 & 1.187 & 0.014 & 0.012 & 13.163 & 8.734 \\
\hline Laairan & 549 & 0.189 & 1.005 & 1.827 & 0.627 & 0.016 & 0.005 & 20.233 & 3.104 \\
\hline Lamiran Cooperation & 520 & 0.171 & 1.004 & 0.707 & 0.825 & 0.008 & 0.011 & 3.654 & 8.010 \\
\hline Leasing Iran & 470 & 0.185 & 1.004 & 3.236 & 1.415 & 0.027 & 0.015 & 31.119 & 13.075 \\
\hline Leasing is self-sufficient & 553 & 0.178 & 1.005 & 1.491 & 1.147 & 0.012 & 0.011 & 17.619 & 6.602 \\
\hline Leasing of industry and mining & 557 & 0.181 & 1.004 & 1.429 & 1.423 & 0.012 & 0.011 & 9.866 & 4.638 \\
\hline Lebanese Calber & 526 & 0.185 & 1.005 & 3.520 & 1.341 & 0.027 & 0.012 & 43.583 & 8.902 \\
\hline Loghman Pharmaceutical & 530 & 0.413 & 0.035 & 2.281 & 1.820 & 0.018 & 0.013 & 27.869 & 15.830 \\
\hline Lotus Parsian Capital & 566 & 0.166 & 1.004 & 1.418 & 0.962 & 0.013 & 0.009 & 9.456 & 5.757 \\
\hline Margarine & 548 & 0.185 & 1.004 & 2.292 & 1.253 & 0.020 & 0.014 & 24.321 & 12.483 \\
\hline Masa Shahid Bahonar & 549 & 0.180 & 1.005 & 3.036 & 0.347 & 0.023 & 0.004 & 26.529 & 1.418 \\
\hline Mashhad construction ring & 565 & 0.180 & 1.006 & 3.623 & 1.531 & 0.027 & 0.014 & 45.910 & 15.913 \\
\hline Medicine: Mystery & 563 & 0.170 & 0.004 & 1.364 & 1.049 & 0.011 & 0.011 & 11.698 & 10.159 \\
\hline Mehrkama Parsa & 563 & 0.182 & 1.004 & 2.786 & 1.348 & 0.021 & 0.014 & 29.844 & 9.894 \\
\hline Melat Bank & 455 & 0.196 & 1.004 & 2.451 & 0.649 & 0.020 & 0.008 & 22.362 & 4.372 \\
\hline Middle East Bank & 569 & 0.160 & 1.004 & 1.595 & 1.746 & 0.013 & 0.012 & 10.449 & 8.732 \\
\hline Middle East Confirmation & 558 & 0.181 & 1.005 & 1.763 & 0.893 & 0.013 & 0.007 & 10.598 & 3.537 \\
\hline Minerals of Iranian salts & 553 & 0.164 & 1.004 & 1.120 & 1.209 & 0.008 & 0.012 & 5.575 & 11.601 \\
\hline Mining, Industry, Tent & 564 & 0.171 & 1.005 & 1.545 & 0.894 & 0.013 & 0.007 & 10.243 & 4.880 \\
\hline Motogen & 561 & 0.173 & 1.004 & 0.995 & 0.890 & 0.010 & 0.011 & 8.056 & 8.870 \\
\hline Motor manufacturers of tractors in Iran & 555 & 0.183 & 1.003 & 3.323 & 1.640 & 0.025 & 0.014 & 36.031 & 8.953 \\
\hline Nation Insurance & 525 & 0.178 & 1.004 & 1.036 & 0.667 & 0.010 & 0.006 & 7.138 & 2.654 \\
\hline National Deve. Group Investment & 569 & 0.171 & 1.004 & 2.533 & 1.490 & 0.019 & 0.015 & 20.971 & 17.121 \\
\hline National Guard of Iran & 572 & 0.183 & 1.005 & 1.806 & 0.865 & 0.013 & 0.009 & 12.505 & 6.989 \\
\hline National Industrial Union of Iran & 563 & 0.171 & 1.004 & 1.413 & 0.976 & 0.011 & 0.008 & 10.874 & 3.400 \\
\hline National Industrial Group & 543 & 0.186 & 0.005 & 0.959 & 1.543 & 0.009 & 0.013 & 4.818 & 5.632 \\
\hline New capital supply & 554 & 0.170 & 1.004 & 1.659 & 1.423 & 0.013 & 0.011 & 9.532 & 8.143 \\
\hline New Fars Cement & 555 & 0.172 & 1.004 & 2.120 & 1.251 & 0.018 & 0.010 & 18.333 & 3.862 \\
\hline Nirookoler & 549 & 0.180 & 1.004 & 2.344 & 1.159 & 0.018 & 0.010 & 17.028 & 11.960 \\
\hline Nishaboor Sugar & 560 & 0.282 & 0.010 & 1.400 & 1.300 & 0.011 & 0.010 & 9.655 & 5.521 \\
\hline Noori Petrochemical & 486 & 1.171 & 1.068 & 2.239 & 2.141 & 0.019 & 0.016 & 15.986 & 13.079 \\
\hline Noosh Mazandaran & 424 & 0.180 & 1.010 & 1.796 & 1.643 & 0.019 & 0.020 & 18.822 & 11.167 \\
\hline North Cement & 560 & 0.174 & 1.004 & 1.570 & 0.847 & 0.013 & 0.006 & 10.819 & 4.160 \\
\hline North Drilling & 538 & 0.188 & 1.004 & 2.919 & 1.156 & 0.022 & 0.012 & 25.222 & 11.536 \\
\hline Northeast Housing Investment & 550 & 0.171 & 1.004 & 3.690 & 0.603 & 0.028 & 0.008 & 41.606 & 3.971 \\
\hline Offset & 558 & 0.177 & 1.003 & 3.216 & 0.996 & 0.026 & 0.011 & 38.601 & 10.790 \\
\hline Ofogh Kourosh chain stores & 556 & 0.161 & 1.004 & 3.028 & 1.536 & 0.023 & 0.013 & 29.064 & 9.822 \\
\hline Omid Investment Management Group & 539 & 0.155 & 1.005 & 2.085 & 0.943 & 0.016 & 0.009 & 13.137 & 3.624 \\
\hline Our Insurance & 566 & 0.166 & 1.005 & 2.570 & 1.499 & 0.023 & 0.012 & 31.366 & 9.488 \\
\hline Paksan & 581 & 0.172 & 1.002 & 2.368 & 1.332 & 0.018 & 0.012 & 18.084 & 9.440 \\
\hline Pars Daroo & 557 & 0.165 & 1.004 & 1.998 & 0.894 & 0.015 & 0.010 & 18.943 & 8.793 \\
\hline
\end{tabular}




\begin{tabular}{|c|c|c|c|c|c|c|c|c|c|}
\hline Name & $\mathbf{N}$ & Volatility & Avg. Return & Kuiper 1D & Kuiper 2D & MAD 1D & MAD 2D & CHIS 1D & CHIS 2D \\
\hline Pars Electric & 547 & 0.194 & 1.006 & 2.274 & 1.478 & 0.023 & 0.011 & 31.342 & 8.540 \\
\hline Pars Tile and Ceramic & 530 & 0.180 & 1.007 & 0.620 & 1.627 & 0.010 & 0.012 & 5.947 & 6.759 \\
\hline Pars Khodro & 531 & 0.189 & 1.004 & 2.970 & 1.264 & 0.024 & 0.012 & 28.188 & 10.617 \\
\hline Pars Minoo & 542 & 0.208 & 1.005 & 2.105 & 1.538 & 0.016 & 0.012 & 18.531 & 12.361 \\
\hline Pars National Agro-industry & 553 & 0.177 & 1.006 & 0.767 & 1.375 & 0.007 & 0.012 & 2.490 & 8.135 \\
\hline Pars Oil & 500 & 0.181 & 1.005 & 1.120 & 0.586 & 0.010 & 0.007 & 5.675 & 3.184 \\
\hline Pars Paper Industries Group & 247 & 0.853 & 1.043 & 2.872 & 1.147 & 0.032 & 0.016 & 28.623 & 6.496 \\
\hline Pars Petrochemical & 564 & 0.155 & 1.004 & 1.619 & 1.183 & 0.013 & 0.013 & 10.831 & 9.864 \\
\hline Pars Saram & 520 & 0.182 & 1.004 & 0.795 & 0.741 & 0.007 & 0.007 & 4.929 & 3.883 \\
\hline Pars Switsch & 522 & 0.198 & 1.006 & 1.808 & 1.210 & 0.014 & 0.014 & 14.923 & 12.029 \\
\hline Parsian Bank & 533 & 0.192 & 1.006 & 1.291 & 1.256 & 0.012 & 0.012 & 8.824 & 8.697 \\
\hline Parsian e-commerce & 562 & 0.176 & 0.003 & 1.095 & 0.852 & 0.012 & 0.010 & 7.890 & 8.025 \\
\hline Parsian Insurance & 565 & 0.175 & 1.005 & 1.517 & 1.395 & 0.011 & 0.009 & 9.244 & 6.061 \\
\hline Parsian Leasing & 270 & 0.368 & 1.011 & 2.564 & 0.783 & 0.033 & 0.011 & 28.366 & 4.891 \\
\hline Pasargad Bank & 556 & 0.165 & 1.005 & 3.451 & 1.252 & 0.026 & 0.014 & 37.280 & 10.792 \\
\hline Pasteur Milk Khorasan & 518 & 0.184 & 1.005 & 2.237 & 2.161 & 0.018 & 0.017 & 18.439 & 20.298 \\
\hline Pasteurized Milk Isfahan Pegah & 550 & 0.169 & 1.004 & 1.405 & 1.201 & 0.011 & 0.017 & 7.949 & 18.033 \\
\hline Pegah of West Azerbaijan & 550 & 0.177 & 1.003 & 2.491 & 1.268 & 0.020 & 0.013 & 21.220 & 6.527 \\
\hline Persian Gulf Energy Mobin & 556 & 0.159 & 1.003 & 0.985 & 1.138 & 0.014 & 0.011 & 12.858 & 9.786 \\
\hline Persian Gulf International Transport & 535 & 0.195 & 1.002 & 2.808 & 0.751 & 0.023 & 0.009 & 28.619 & 5.477 \\
\hline Persian Gulf Petrochemical Industries & 557 & 0.168 & 1.004 & 1.487 & 0.867 & 0.012 & 0.009 & 11.077 & 4.863 \\
\hline Persian refractory products & 469 & 0.188 & 1.006 & 1.188 & 0.874 & 0.013 & 0.011 & 9.429 & 9.520 \\
\hline Petrochemical Bu Ali Sina & 174 & 1.751 & 1.236 & 1.705 & 1.173 & 0.023 & 0.020 & 10.629 & 11.692 \\
\hline Petrochemical Campus & 579 & 0.164 & 1.005 & 2.689 & 1.004 & 0.020 & 0.011 & 26.254 & 10.342 \\
\hline Petrochemical Group S. Iranians & 515 & 0.236 & 1.006 & 1.590 & 0.788 & 0.014 & 0.009 & 11.564 & 5.893 \\
\hline Petrochemical Jam & 538 & 0.159 & 1.004 & 0.606 & 1.250 & 0.007 & 0.010 & 8.633 & 8.970 \\
\hline Petrochemical Kharkiv & 534 & 0.162 & 1.003 & 1.994 & 1.034 & 0.016 & 0.009 & 21.219 & 3.754 \\
\hline Petrochemical Shiraz & 573 & 0.162 & 1.005 & 2.448 & 1.217 & 0.019 & 0.010 & 24.075 & 8.388 \\
\hline Petrochemical Technologists & 552 & 0.161 & 1.005 & 1.981 & 0.840 & 0.017 & 0.012 & 13.763 & 7.945 \\
\hline Petrochemical Transportation \& Log. & 518 & 0.179 & 1.005 & 3.542 & 2.773 & 0.028 & 0.022 & 46.576 & 24.020 \\
\hline Pharma Laboratory Dr. Abidie & 561 & 0.161 & 1.002 & 3.719 & 1.423 & 0.028 & 0.011 & 40.554 & 10.381 \\
\hline Pharmaceutical poisoning & 551 & 0.296 & 0.009 & 2.616 & 0.563 & 0.022 & 0.007 & 33.476 & 3.122 \\
\hline Pharmacist (Holding) & 568 & 0.221 & 0.007 & 1.202 & 0.775 & 0.010 & 0.008 & 6.773 & 4.188 \\
\hline Pharmacy Abu Rihanna & 561 & 0.175 & 0.004 & 1.509 & 1.370 & 0.011 & 0.012 & 9.257 & 9.597 \\
\hline Pharmacy Day of Medicine & 563 & 0.163 & 1.004 & 2.579 & 1.315 & 0.019 & 0.009 & 30.360 & 4.967 \\
\hline Pharmacy Elixir & 572 & 0.167 & 0.005 & 1.540 & 0.894 & 0.012 & 0.011 & 10.104 & 5.777 \\
\hline Pharmacy Farabi & 552 & 0.261 & 0.008 & 4.517 & 0.871 & 0.035 & 0.010 & 58.095 & 7.994 \\
\hline Pharmacy Jabrabana Hayyana & 551 & 0.258 & 0.008 & 2.031 & 1.047 & 0.016 & 0.015 & 16.841 & 16.315 \\
\hline Pharmacy Kowsar & 542 & 0.343 & 0.017 & 2.393 & 0.700 & 0.023 & 0.008 & 33.556 & 5.619 \\
\hline Phoenix & 524 & 0.426 & 1.011 & 2.411 & 1.159 & 0.019 & 0.011 & 27.456 & 7.155 \\
\hline Piazer culture and industry & 546 & 0.183 & 1.005 & 2.118 & 1.235 & 0.016 & 0.013 & 18.455 & 6.774 \\
\hline Plaskokar & 573 & 0.171 & 1.004 & 2.786 & 1.026 & 0.022 & 0.009 & 32.303 & 7.452 \\
\hline Polypropylene Jam - Jam Pollen & 521 & 0.971 & 1.045 & 1.921 & 0.761 & 0.016 & 0.008 & 17.565 & 6.080 \\
\hline Post Bank of Iran & 567 & 0.183 & 1.005 & 2.593 & 0.729 & 0.021 & 0.008 & 24.940 & 3.755 \\
\hline
\end{tabular}




\begin{tabular}{|c|c|c|c|c|c|c|c|c|c|}
\hline Name & $\mathbf{N}$ & Volatility & Avg. Return & Kuiper 1D & Kuiper 2D & MAD 1D & MAD 2D & CHIS 1D & CHIS 2D \\
\hline Power trans & 523 & 0.174 & 1.005 & 1.642 & 2.356 & 0.013 & 0.017 & 12.215 & 16.329 \\
\hline Produced by Mehram & 532 & 0.174 & 1.004 & 0.857 & 0.982 & 0.012 & 0.012 & 10.708 & 5.703 \\
\hline Produced by Taksim & 538 & 0.190 & 1.004 & 1.776 & 1.776 & 0.014 & 0.017 & 13.513 & 17.542 \\
\hline Production of cast iron makers & 537 & 0.184 & 1.005 & 0.568 & 0.648 & 0.007 & 0.009 & 3.611 & 6.440 \\
\hline Production of raw materials & 569 & 0.182 & 1.005 & 2.692 & 1.144 & 0.020 & 0.011 & 21.194 & 6.941 \\
\hline Products of food and sugarcane & 541 & 0.147 & 1.004 & 1.333 & 0.993 & 0.013 & 0.007 & 9.147 & 6.371 \\
\hline Qazvin sugar factories & 548 & 0.184 & 1.003 & 3.220 & 0.933 & 0.025 & 0.010 & 29.795 & 6.276 \\
\hline Radiator Iran & 552 & 0.201 & 1.007 & 0.937 & 0.928 & 0.013 & 0.006 & 8.532 & 3.486 \\
\hline Ramina & 558 & 0.180 & 1.004 & 1.268 & 0.695 & 0.012 & 0.009 & 9.170 & 5.581 \\
\hline Rena Investment (Holding) & 569 & 0.182 & 1.004 & 1.921 & 0.904 & 0.015 & 0.009 & 17.821 & 5.282 \\
\hline Renovation Construction Tehran & 552 & 0.184 & 1.005 & 3.573 & 0.455 & 0.027 & 0.006 & 42.155 & 3.211 \\
\hline Retirement of bank employees & 566 & 0.165 & 1.004 & 1.889 & 1.056 & 0.019 & 0.008 & 21.317 & 7.120 \\
\hline Rolled aluminum & 563 & 0.176 & 1.004 & 1.188 & 1.436 & 0.013 & 0.012 & 10.059 & 13.472 \\
\hline Rollers of Sepahan & 471 & 0.600 & 0.035 & 1.225 & 1.257 & 0.010 & 0.013 & 7.872 & 13.761 \\
\hline Rolling and Steel Parts & 564 & 0.159 & 1.006 & 1.493 & 0.944 & 0.012 & 0.008 & 9.911 & 5.549 \\
\hline Rotary & 556 & 0.177 & 1.004 & 1.783 & 0.755 & 0.013 & 0.008 & 8.901 & 2.636 \\
\hline Industrial Chemicals & 551 & 0.180 & 1.005 & 1.290 & 0.792 & 0.010 & 0.009 & 12.025 & 6.405 \\
\hline Sabanour Mining and Industrial Dev. & 554 & 0.170 & 1.006 & 3.231 & 0.773 & 0.024 & 0.010 & 34.689 & 5.762 \\
\hline Sadi Tile and Ceramic & 537 & 0.191 & 1.004 & 2.212 & 1.493 & 0.017 & 0.013 & 16.828 & 11.033 \\
\hline Sadr Tamin Investment & 546 & 0.241 & 1.007 & 2.629 & 0.828 & 0.020 & 0.009 & 20.916 & 7.164 \\
\hline Sahand Rubber Industry & 568 & 0.175 & 1.004 & 2.928 & 2.046 & 0.022 & 0.014 & 29.514 & 15.706 \\
\hline Saipa & 527 & 0.235 & 1.005 & 2.538 & 0.713 & 0.020 & 0.009 & 26.900 & 5.284 \\
\hline Saipa Azin & 568 & 0.182 & 1.002 & 3.609 & 0.652 & 0.027 & 0.008 & 38.139 & 5.182 \\
\hline Saipa Computer Leasing & 564 & 0.176 & 1.002 & 1.622 & 0.998 & 0.012 & 0.010 & 8.099 & 8.192 \\
\hline Saipa Investment & 563 & 0.179 & 1.004 & 1.788 & 1.089 & 0.018 & 0.011 & 24.721 & 9.949 \\
\hline Salamin & 555 & 0.191 & 1.004 & 1.792 & 1.556 & 0.015 & 0.011 & 14.158 & 7.451 \\
\hline Saman Kish electronic payment & 566 & 0.167 & 1.003 & 0.766 & 1.464 & 0.007 & 0.012 & 4.237 & 7.751 \\
\hline Sarma Afarin & 559 & 0.177 & 1.005 & 2.862 & 1.269 & 0.022 & 0.011 & 25.395 & 8.316 \\
\hline Securing Cement Investment & 274 & 0.944 & 1.055 & 2.888 & 2.056 & 0.033 & 0.022 & 28.575 & 15.881 \\
\hline Sepahan Industrial Group & 566 & 0.179 & 1.005 & 1.098 & 1.012 & 0.010 & 0.009 & 7.207 & 6.333 \\
\hline Sepanta Communication & 571 & 0.158 & 1.005 & 0.926 & 1.288 & 0.007 & 0.012 & 4.324 & 6.973 \\
\hline Shahd & 538 & 0.154 & 1.002 & 2.529 & 2.066 & 0.019 & 0.022 & 23.231 & 30.142 \\
\hline Shahd Iran & 543 & 0.187 & 1.003 & 1.274 & 1.052 & 0.010 & 0.007 & 7.764 & 5.781 \\
\hline Shahid Ghandi Production Factories & 518 & 0.193 & 1.006 & 1.252 & 1.181 & 0.013 & 0.011 & 10.126 & 8.437 \\
\hline Shazand Petrochemical & 577 & 0.170 & 1.004 & 1.437 & 1.485 & 0.012 & 0.011 & 11.328 & 8.107 \\
\hline Shipping of the Islamic Republic of Iran & 549 & 0.184 & 1.003 & 0.352 & 0.724 & 0.006 & 0.007 & 2.131 & 4.019 \\
\hline Siman Behbahan & 553 & 0.184 & 1.004 & 1.157 & 0.785 & 0.011 & 0.009 & 11.813 & 6.384 \\
\hline Siman Bojnoord & 565 & 0.179 & 1.003 & 1.491 & 0.755 & 0.015 & 0.008 & 19.474 & 4.171 \\
\hline Siman Darb & 569 & 0.174 & 1.005 & 2.786 & 0.590 & 0.021 & 0.008 & 27.377 & 4.895 \\
\hline Siman Dorood & 558 & 0.194 & 1.004 & 2.545 & 1.654 & 0.019 & 0.014 & 32.969 & 15.476 \\
\hline Siman Fars & 503 & 0.187 & 1.005 & 0.756 & 2.518 & 0.006 & 0.020 & 2.950 & 9.250 \\
\hline Siman Ghaen & 560 & 0.172 & 1.004 & 1.520 & 1.296 & 0.011 & 0.013 & 10.033 & 12.948 \\
\hline Siman Heghmatan & 548 & 0.174 & 1.005 & 0.850 & 1.617 & 0.007 & 0.015 & 3.250 & 16.181 \\
\hline Siman Hormozghan & 532 & 0.157 & 1.004 & 2.249 & 0.674 & 0.017 & 0.009 & 15.106 & 5.775 \\
\hline
\end{tabular}




\begin{tabular}{|c|c|c|c|c|c|c|c|c|c|}
\hline Name & $\mathbf{N}$ & Volatility & Avg. Return & Kuiper 1D & Kuiper 2D & MAD 1D & MAD 2D & CHIS 1D & CHIS 2D \\
\hline Siman Ilam & 543 & 0.196 & 1.004 & 1.901 & 0.614 & 0.017 & 0.009 & 20.115 & 6.501 \\
\hline Siman Isfahan & 530 & 0.191 & 1.007 & 0.968 & 1.425 & 0.010 & 0.013 & 6.823 & 10.908 \\
\hline Siman Kerman & 566 & 0.168 & 1.003 & 3.147 & 1.560 & 0.024 & 0.012 & 32.434 & 10.204 \\
\hline Siman Kordestan & 558 & 0.179 & 1.004 & 2.440 & 1.354 & 0.018 & 0.011 & 28.304 & 13.129 \\
\hline Siman Mazandaran & 573 & 0.177 & 1.004 & 2.718 & 0.552 & 0.020 & 0.008 & 21.800 & 4.556 \\
\hline Siman Sepahan & 574 & 0.176 & 1.004 & 1.691 & 0.936 & 0.013 & 0.010 & 11.364 & 6.017 \\
\hline Siman Shahrood & 565 & 0.173 & 1.005 & 1.320 & 0.633 & 0.010 & 0.008 & 9.530 & 5.726 \\
\hline Siman Soofian & 558 & 0.239 & 1.006 & 1.475 & 0.698 & 0.012 & 0.012 & 11.212 & 9.055 \\
\hline Siman Urumieh & 564 & 0.181 & 1.005 & 2.962 & 0.705 & 0.022 & 0.008 & 36.948 & 4.593 \\
\hline Sina Pharmacy & 566 & 0.254 & 0.005 & 1.846 & 0.960 & 0.014 & 0.007 & 10.548 & 4.647 \\
\hline Sinai Chemical Industry & 539 & 0.184 & 1.007 & 2.211 & 1.023 & 0.017 & 0.010 & 16.254 & 7.638 \\
\hline Sinai Tile and Ceramic Industry & 519 & 0.179 & 1.006 & 3.108 & 1.210 & 0.024 & 0.012 & 31.307 & 12.887 \\
\hline Sobhan Daru & 553 & 0.261 & 0.008 & 3.189 & 1.013 & 0.024 & 0.008 & 33.441 & 5.775 \\
\hline Sobhan Pharmaceutical Group & 561 & 0.169 & 0.004 & 2.724 & 1.023 & 0.021 & 0.013 & 28.884 & 10.050 \\
\hline Steel of Khuzestan & 569 & 0.168 & 0.004 & 2.575 & 1.016 & 0.019 & 0.011 & 21.987 & 7.266 \\
\hline Sugar Hekmatan & 546 & 0.180 & 1.003 & 1.799 & 1.172 & 0.015 & 0.014 & 14.985 & 10.537 \\
\hline Sugar Isfahan & 570 & 0.236 & 0.006 & 1.505 & 0.887 & 0.013 & 0.012 & 10.324 & 7.948 \\
\hline Sugar Lorestan & 528 & 0.169 & 0.003 & 4.173 & 0.700 & 0.032 & 0.009 & 60.935 & 5.118 \\
\hline Sugar Mardoosht & 533 & 0.184 & 0.005 & 1.002 & 1.645 & 0.011 & 0.012 & 6.856 & 10.853 \\
\hline Sugar Shahrood & 548 & 0.182 & 1.005 & 2.773 & 0.946 & 0.021 & 0.009 & 35.235 & 4.426 \\
\hline Supply of spilled sand & 503 & 0.188 & 1.004 & 1.440 & 0.866 & 0.012 & 0.012 & 13.759 & 9.445 \\
\hline System Partners & 541 & 0.158 & 1.004 & 0.475 & 1.094 & 0.007 & 0.012 & 4.669 & 9.823 \\
\hline Tabahsar & 246 & 0.287 & 1.006 & 2.063 & 0.812 & 0.029 & 0.011 & 23.549 & 5.906 \\
\hline Tabriz Oil Refinery & 559 & 0.177 & 1.004 & 0.715 & 1.000 & 0.010 & 0.012 & 6.768 & 12.395 \\
\hline Tamin Oil, Gas and Petrochemical & 544 & 0.174 & 1.004 & 1.453 & 1.533 & 0.013 & 0.014 & 10.106 & 13.541 \\
\hline Tamin Pharmaceutical Investment & 557 & 0.226 & 0.007 & 1.809 & 1.448 & 0.016 & 0.013 & 21.633 & 6.893 \\
\hline Tehran Oil Refinery & 544 & 0.184 & 1.004 & 3.664 & 0.846 & 0.028 & 0.008 & 44.181 & 3.275 \\
\hline Tehran Siman & 544 & 0.191 & 1.004 & 3.135 & 1.228 & 0.024 & 0.010 & 41.540 & 8.903 \\
\hline Teknotar & 546 & 0.182 & 1.004 & 4.638 & 1.715 & 0.035 & 0.012 & 82.489 & 9.925 \\
\hline The cement is white & 546 & 0.172 & 1.005 & 1.573 & 2.073 & 0.012 & 0.014 & 9.078 & 13.373 \\
\hline Iranian Tractor Manufacturer & 551 & 0.179 & 1.005 & 1.852 & 1.714 & 0.014 & 0.012 & 17.347 & 13.626 \\
\hline The dairy is clean & 534 & 0.229 & 1.005 & 3.277 & 1.022 & 0.025 & 0.012 & 42.914 & 13.508 \\
\hline The food of the Persian dam & 533 & 0.174 & 1.005 & 0.888 & 0.879 & 0.007 & 0.009 & 3.846 & 6.779 \\
\hline The glass of Hamedan & 569 & 0.177 & 1.004 & 1.705 & 0.642 & 0.013 & 0.006 & 10.038 & 3.615 \\
\hline The lamp of the Persian Meteor & 540 & 0.175 & 1.006 & 2.502 & 1.545 & 0.020 & 0.011 & 20.354 & 9.130 \\
\hline The Medicine Cup & 516 & 0.170 & 1.004 & 1.311 & 0.783 & 0.012 & 0.008 & 11.899 & 4.849 \\
\hline Sugar Khorasan & 452 & 0.200 & 1.007 & 1.388 & 0.816 & 0.014 & 0.010 & 11.601 & 4.815 \\
\hline Tile and Ceramic Memory & 532 & 0.196 & 1.006 & 2.262 & 0.782 & 0.018 & 0.006 & 23.216 & 2.203 \\
\hline To pay the nation & 562 & 0.163 & 1.003 & 2.808 & 1.011 & 0.021 & 0.008 & 23.865 & 2.791 \\
\hline Tourism and Welfare of Abadgaran Iran & 545 & 0.166 & 1.006 & 2.410 & 1.900 & 0.018 & 0.013 & 21.527 & 13.017 \\
\hline Traktorsazi Iran & 558 & 0.172 & 1.005 & 0.754 & 0.684 & 0.007 & 0.012 & 6.393 & 12.721 \\
\hline Transportation Toka & 557 & 0.190 & 1.005 & 4.217 & 0.686 & 0.032 & 0.009 & 73.389 & 6.161 \\
\hline Urban Development & 553 & 0.173 & 1.005 & 4.571 & 0.971 & 0.035 & 0.008 & 64.377 & 5.747 \\
\hline Vaspari Mellat & 567 & 0.183 & 1.004 & 0.961 & 1.340 & 0.007 & 0.011 & 5.124 & 9.782 \\
\hline
\end{tabular}




\begin{tabular}{|c|c|c|c|c|c|c|c|c|c|}
\hline Name & $\mathbf{N}$ & Volatility & Avg. Return & Kuiper 1D & Kuiper 2D & MAD 1D & MAD 2D & CHIS 1D & CHIS 2D \\
\hline Web-based data spreaders & 495 & 0.182 & 1.003 & 2.542 & 0.788 & 0.020 & 0.008 & 28.802 & 3.752 \\
\hline West Siman & 561 & 0.167 & 1.004 & 1.987 & 1.191 & 0.015 & 0.013 & 17.523 & 10.980 \\
\hline White Farab Kavir Steel Production & 430 & 0.505 & 1.018 & 2.885 & 1.311 & 0.025 & 0.013 & 26.813 & 9.850 \\
\hline With the help of technology & 557 & 0.184 & 1.005 & 1.952 & 0.598 & 0.016 & 0.009 & 17.321 & 5.849 \\
\hline Witness investment & 564 & 0.179 & 1.005 & 0.707 & 1.458 & 0.006 & 0.012 & 3.417 & 11.339 \\
\hline Zagros Pharmacy Pars Pharmacy & 559 & 0.180 & 0.004 & 3.126 & 0.802 & 0.024 & 0.010 & 35.278 & 8.311 \\
\hline Zamyad & 530 & 0.184 & 1.005 & 2.809 & 1.545 & 0.022 & 0.017 & 25.818 & 19.148 \\
\hline
\end{tabular}

Notes. MAD: Median Average Deviation; CHIS: Chi-Square; 1D = Fist-Digit; 2D = Second-Digit.

Table 4. Results of the empirical tests

\begin{tabular}{lllllll}
\hline Conformity & Chi-Square & & Kuiper & & MAD & 2nd digit \\
& 1st digit & 2nd digit & 1st digit & 2nd digit & 2nd dit \\
\hline Yes & 149 & 17 & 190 & 116 & 134 & 4 \\
No & 192 & 324 & 151 & 225 & 207 & 337 \\
\hline
\end{tabular}

\section{Discussion}

A thorough evaluation of intraday demand for public shares listed on the TSE revealed significant noncompliance with Benford's Law. There is overwhelming evidence that shares listed on the TSE are most likely to be affected by attempts to manipulate the market, most likely by a falsified number of intraday trades reported by the Iranian Financial Market Regulatory Authorities. The most common BL violations in this study were observed in various industries. Companies across the mining, financial services, banking, construction, pharmaceutical, energy, and manufacturing sectors were equally identified among the most frequent $\mathrm{BL}$ violators.

As mentioned, Iran's supreme leader, Ali Khamenei, repeatedly encouraged citizens and called for proactive engagement in investing in the country's primary and secondary equity markets. Analysts claim that these attempts were primarily associated with the desolate Iranian economy. Moreover, the persistent mismanagement, continuous incompetence, bribery in public administration, and international political and economic pressures on Iran have left deep scars on the Iranian economy (Bertelsmann Stiftung, 2020).

Iran is indeed a developing country that can barely sustain its economy on its own.

State-owned enterprises (SOEs) account for approximately $80 \%$ of the country's economic activity. According to the International Monetary Fund (IMF), as of December 2020, public sector debt exceeded IRR 500 trillion. More than $89 \%$ was held by the theocratic government and less than $11 \%$ by state-owned enterprises (IMF, 2020). Iran's national debt has increased by $41 \%$ annually over the past ten years. In terms of the soundness of its banking system, Iran ranks 131 out of 140 worldwide. These facts can explain Iran's political leadership motivation to encourage capital inflow through people's engagement in the TSE transactions. Figure 3 highlights Iran's national debt as forecasted by International Monetary Fund (IMF) until 2025.

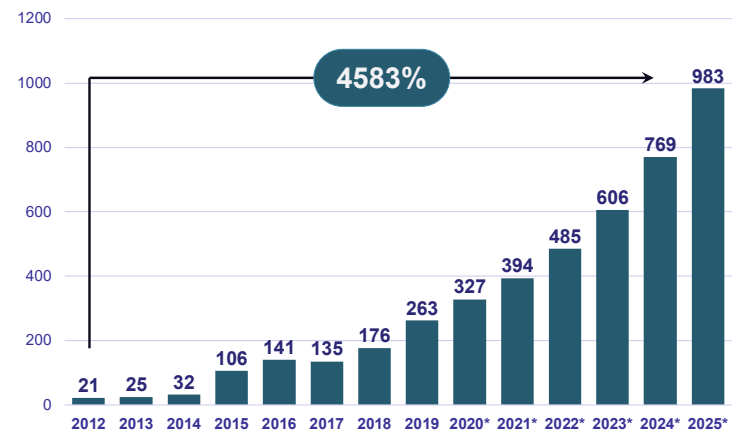

Figure 3. IMF forecasts for Iranian national debt until 2025

To plug budget holes, the clerical regime has repeatedly raised (latent) taxes, leading to widespread public unrest 
and scrutiny. With Initial Public Offerings (IPO) throughout semi-functioning equity markets, the Islamic regime was able to access the people's savings and capital that had less engagement in Iran's equity markets. The deficit is indeed an additional motive for the interventions of the government across the TSE. It can be a driver behind the questionable development of the securities. Despite the impact of the U.S. sanctions on the Iranian crude oil exports, the country is a paragon of profligacy. For example, the Islamic government's annual budget for 2020/21 included a substantial $\$ 40$ million for the domestic travel expenses of the supreme leader, Ali Khamenei (Iran Budget, 2021). Comparing this amount to the $\$ 14$ million travelling costs of the former U.S. President Barack Obama (Delikik, 2020) shows how wasteful and inefficient the government in Iran is. Accordingly, Khamenei received three times more taxpayer money for his national trips than the former U.S. president for his international presence. This is quite surprising, as the chief cleric has already banned "too many foreign trips" by Iranian officials to save foreign currency in 2018 (Radio Farda, 2018).

Like budgeting and planning practices in Iran, the TSE is opaque. The lack of data integrity in Iran is nothing new. One can assume that the efficient market hypothesis cannot function in Iran due to the low quality of data. Existing investors in Iran shall exercise extreme caution when trading on TSEs. Given the empirical evidence found in this study, it is imperative to perform due diligence on all securities listed on TSE, including financial statements, the number of intraday transactions, representing transaction value and volume. This way, Iranian investors would be better able to navigate through the inherent risks of untransparent transactions on TSE. Nonetheless, novice investors should refrain from making any new transactions on the TSE until the market data is proven to be conclusive and reliable.

\subsection{Limitations}

This study is based mainly on the Tehran State Stock Exchange data. Former studies on the financial market in Iran do not exist. The literature driven by Iranian scientists appears to be inconclusive and compliant with the political will of the Iranian government. Given the lack of prior knowledge in the literature in this context, we took due care to avoid cognitive biases such as naïve realism, conformation, and authority biases while studying the literature and collecting data.

\subsection{Future Research}

In view of our results, we suggest that the actual returns and risks of the best-performing stocks should be examined based on the portfolio selection theory. Although we recognize the overall issues with financial data in Iran, in a hypothetical investment scenario, one should determine the efficient frontier portfolio. This would greatly enhance the present understanding of the TSE indices as a multi-layered and diversified financial market and provide some guidance to investors who are quite inexperienced with the advanced techniques of portfolio management.

\section{References}

Aggarwal, V., \& Dharni, K. (2020). Deshelling the Shell Companies Using Benford's Law: An Emerging Market Study. Vikalpa, 45(3), 160-169. https://doi.org/10.1177/0256090920979695

Beneish, M. D. (1999). The detection of earnings manipulation. Financial Analysts Journal, 55(5), 24-36.

Benford, F. (1938). The law of anomalous numbers. Proceedings of the American Philosophical Society, 78, 551572 .

Bertelsmann Stiftung. (2020). Bertelsmann Transformation Index (BTI) for Iran. Retrieved from https://btiproject.org/en/?\&cb=00000

Carswell R. (1981). Economic sanctions and the Iran experience. Foreign Affairs, 60(2), 247-265. https://doi.org/10.2307/20041079

Corazza, M., Ellero, A., \& Zorzi, A. (2010). Checking financial markets via Benford's law: the S\&P 500 case. In M. Corazza and C. Pizzi (Eds.), Mathematical and statistical methods for actuarial sciences and finance (pp. 93-102). Springer, Italia.

Deleanu, I. S. (2017). Do countries regularly misinform the international community about their anti-money laundering efforts? Evidence Using Benford's Law. PLOS ONE, 12(1), e0169632. https://doi.org/10.1371/journal.pone.0169632

Delkic, M. (2017). Obama Administration Travel Cost $\$ 114$ Million, Trump's So Far Is \$10 Million. Retrieved from https://www.newsweek.com/obama-administration-travel-costs-114-million-trump-723883

Durtschi, C., Hillison, W, \& Pacini, C. (2004). The effective use of Benford's law to assist in detecting fraud in accounting data. JFAR, 17(34), 18. 
Fama, E. (1963). Mandelbrot and the stable paretian hypothesis. Journal of Business, 36, 420-429.

Fama, E. (1965a). The behavior of stock prices. Journal of Business, 38, 34-105.

Fama, E. (1965b). Random walks in stock market prices. Financial Analysts Journal, 21, 55-59.

Fama, E. (1970). Efficient capital markets: a survey of theory and empirical work. Journal of Finance, 25, 383417.

Farhadi N., \& Lahooti, H. (2021b). Pandemic Growth and Benfordness: Empirical Evidence from 176 Countries Worldwide. COVID, 1(1), 366-383. https://doi.org/10.3390/covid1010031

Farhadi, N. (2021). Can we rely on COVID-19 data? An assessment of data from over 200 countries. Science Progress, 104(2), 1-19.

Farhadi, N., \& Lahooti, H. (2021a). Are COVID-19 data reliable? A quantitative analysis of pandemic data from 182 countries. COVID, 1(1), 137-152. https://doi.org/10.3390/covid1010013

Finnerty, J. D (2005). Short selling, death spiral convertibles, and the profitability of stock manipulation, Retrieved from https://www.sec.gov/comments/s7-08-08/s70808-318.pdf

Fox, M. B. (2018). Stock market manipulation and its regulation. Yale J. Reg. 35(1), 67-126.

Goudarzi, H. (2014). Stock Market Volatility under Sanctions. International Journal of Management and Sustainability, 3(4), 234-249.

Grammatikos, T., \& Papanikolaou, N. I. (2020). Application of Benford's law to detect accounting manipulation in the banking sector. Journal of Financial Services Research. https://doi.org/10.1007/s10693-020-00334-9

Hauzen. (2021). Market Manipulation. Retrieved from https://hauzen.hk/market-manipulation

International Monetary Fund. (2020). The Islamic Republic of Iran. Retrieved from https://www.imf.org/en/Countries/IRN. Last accessed 28/9/2021

Iran Budget. (2020). Retrieved from https://iranbudget.org/expenses1399/1399/1/b77bc0e2

Iran International News. (2021). Survey shows 77 percent of investors lost in Tehran stock market. Retrieved from https://www.iranintl.com/en/iran-in-brief/survey-shows-77-percent-investors-tehran-stock-market-lost

Karavardar, A. (2014). Benford's Law and an Analysis in Istanbul Stock Exchange (BIST). International Journal of Business and Management, 9(4). https://doi.org/10.5539/ijbm.v9n4p160

Khaasteh, R. (2021). Iran experiences millionaire boom despite US sanctions and fifth Covid 19 wave. Retrieved from https://www.middleeasteye.net/news/iran-millionaire-boom-us-sanctions-covid-pandemic

Lomnicka, E. (2001). Preventing and Controlling the Manipulation of Financial Markets: Towards a Definition of 'Market Manipulation. Journal of Financial Crime, 8(4), 297-304. https://doi.org/10.1108/eb025994

Luna, D. K., Palshikar, G. K., Apte, M., \& Bhattacharya, A. (2018). Finding shell company accounts using anomaly detection. In Proceedings of the ACM India Joint International Conference on Data Science and Management of Data, 18, 167-174.

MacCarthy, J. (2017). Using Altman Z-score and Beneish M-score models to detect financial fraud and corporate failure. International Journal of Finance and Accounting, 6(6), 159-166.

Markowitz, H. (1952). Portfolio selection. Journal of Finance, 7, 77-91.

Nasdaq. (2020). Five market manipulation tactics and how to avoid them. Retrieved from https://www.nasdaq.com/articles/5-market-manipulation-tactics-and-how-avoid-them-2018-04-11

Nasdaq. (2021). Wash Trading: Frequently Asked Questions. Retrieved from https://www.nasdaq.com/docs/98477_wash-trading-faq-.pdf

Newcomb, S. (1881). Note on the Frequency of Use of the Different Digits in Natural 242 Numbers. American Journal of Mathematics, 4(1), 39-40.

Nigrini, M. (1996). A taxpayer compliance application of Benford's law. Journal of the American Taxation Association, 18, 72-91.

Nigrini, M. (2000). Digital Analysis Using Benford's Law. Global Audit Publications, Vancouver, B.C., Canada.

Nigrini, M. (2012). Benford's law: applications for forensic accounting, auditing and fraud detection. New York: John Wiley and Sons. 
Nigrini, M., \& Mittermaier, L. J. (1997). The use of Benford's law as an aid in analytical procedures. Auditing: A Journal of Practice and Theory, 16(Fall), 52-67.

Radio, F. (2018). Khamenei Forbids' Too Much Travelling Abroad' To Save Foreign Currency. Retrieved from: https://en.radiofarda.com/a/khamenei-forbids-too-much-travelling-abroad-to-save-foreigncurrency/29292161.html

Ravenda, D., Valencia-Silva, M. M., Argiles-Bosch, J. M., \& Garcia-Blandon, J. (2018). Accrual management as an indication of money laundering through legally registered Mafia firms in Italy. Accounting, Auditing \& Accountability Journal, 31(1). 286-317.

Rome, H. (2021). Iran in 2021: The Economy. Retrieved fromhttps://iranprimer.usip.org/blog/2021/dec/15/iran2021-economy

Roukema, B. F. (2014). A first-digit anomaly in the 2009 Iranian presidential election. J Appl Stat., 41, 164-199.

Sambridge, M., \& Jackson, J. (2020). National COVID figures - Benford's law looks for errors. Retrieved from: https://www.nature.com/articles/d41586-020-01565-5

Shengmin, Z., \& Wenchao, W. (2010). Does Chinese stock indices agree with Benford's law? 2010 International Conference on Management and Service Science.

Stack, G. (2015). Shell companies, Latvian-type correspondent banking, money laundering and illicit financial flows from Russia and the former Soviet Union. Journal of Money Laundering Control, 18(4), 496-512.

Tehran Stock Exchange (TSE). (2021b). Retrieved from CEICDATA.com

Tehran Stock Exchange. (2021). History. Retrieved from https://tse.ir/cms/Page56.html

Thomson Reuters. (2020). Spoofing' in the marketplace: a comparison of US and UK legislation and enforcement. Retrieved from https://www.kslaw.com/attachments/000/007/103/original/Spoofing_the_market_a_comparison_of_US_and _UK_law_and_enforcement.pdf

U.S. Securities and Exchange Commission. (2020). Market Manipulation. Retrieved from https://www.investor.gov/introduction-investing/investing-basics/glossary/market-manipulation

Williams, O. A. (2021). Iran sees millionaire boom amid sanctions, Covid-19 and an election. Retrieved from https://www.forbes.com/sites/oliverwilliams1/2021/06/28/iran-sees-millionaire-boom-amid-sanctionscovid-19-and-an-election/?sh=767b901 fa93b

Yazdani, S. (2008). Financial market development and agricultural economic growth in Iran. American-Eurasian Journal of Sustainable Agriculture, 2(3), 338-343.

\section{Copyrights}

Copyright for this article is retained by the author(s), with first publication rights granted to the journal.

This is an open-access article distributed under the terms and conditions of the Creative Commons Attribution license (http://creativecommons.org/licenses/by/4.0/). 